\title{
The study of the elastic characteristics of carbon fiber with a 3D structure
}

\author{
V.S. Zhernakov, Y.S. Pervushin, P. V. Solovyev ${ }^{\dagger}$ \\ †paulnightingale@mail.ru
}

FSBEO HE “Ufa State Aviation Technical University”, 12 Karl Marks St., Ufa, 450008, Russia

Composite materials are now widely used in aircraft manufacturing, rocket production, shipbuilding, the petroleum and chemical industries. Multilayer composites are the most promising from the viewpoint of controlling their physical and mechanical properties, depending on the direction of external forces during operation. By changing the multilayer composite structure (the number of layers, the angles and the sequence of their stacking, the physical and mechanical properties of the composite components), it is possible to control their physical and mechanical characteristics in a given direction. The article presents the results of an analytical, experimental and finite-element exploration of the influence of a unidirectional spatial reinforcement of carbon fiber composite with cross-piercing on its elastic compliance coefficients and strength characteristics. A literature review of methods for determining the elastic characteristics of composite materials (CM) with 3D (spatial) structures is given. Methods of determining elastic characteristics of spatially reinforced composites described in the literature are based on the principle of splitting them into layers, in which a unidirectional layer characterized by nine elastic parameters is taken as the basis: three elastic moduli along the main axes of elastic symmetry $E_{1}, E_{2}, E_{3}$, three shear moduli $G_{12}, G_{23}, G_{13}$ and three Poisson coefficients $v_{12}, v_{23}, v_{13}$. From the analysis of the literature sources, it has been established that in determining the compliance coefficients of spatially reinforced composite materials, only the volume fraction of the reinforcing material and its elastic characteristics are taken into account. Studies of the stress-strain state of the matrix modified with a piercing thread showed that its elastic characteristics, in addition to the piercing thread volume content, were influenced by the geometrical parameters of the insertion, the elastic characteristics of the piercing thread impregnated with the matrix material, and the stress concentration on its boundary. This influence occurs in all ways of creating spatial structures formed by a system of three threads. The article also presents the results of the experimental determination of the spatially reinforced CM elastic characteristics and its comparison with the theoretical components of the elastic compliance tensor of a unidirectionally reinforced CM.

Keywords: composite, modified matrix, stitching, 3D structure.

УДК: $620.172 / .178 .2$

\section{Исследование упругих характеристик углепластика с 3D структурой}

\author{
Жернаков В. С., Первушин Ю.С., Соловьев П. В. ${ }^{\dagger}$ \\ ФГБОУ ВО «Уфимский государственный авиационный технический университет», \\ ул. Карла Маркса, 12, Уфа, 450008, Россия
}

Композиционные материалы в настоящее время широко используются в авиастроении, ракетостроении, кораблестроении, нефтяной и химической промышленности. Многослойные композиты являются наиболее перспективными с точки зрения управления их физико-механическими свойствами в зависимости от направления внешних силовых воздействий в процессе эксплуатации. Изменяя многослойную структуру композита (число слоев, углы и последовательность их укладки, физико-механические свойства компонент композита), можно управлять их физико-механическими характеристиками в нужном направлении. В статье приведены результаты аналитического, экспериментального и конечно-элементного исследования влияния пространственного армирования однонаправленного углепластика с поперечной прошивкой на его коэффициенты упругой податливости и прочностные характеристики. Приведен обзор литературных источников, посвященных методам определения 
упругих характеристик композиционных материалов (КМ) с 3D (пространственными) структурами. В основе приведенных в литературе методов определения упругих характеристик пространственно-армированных композитов лежит принцип разбиения их на слои, в которых за основу принимается однонаправленный слой, характеризующийся девятью упругими параметрами: тремя модулями упругости вдоль главных осей упругой симметрии $E_{1}, E_{2}, E_{3}$, тремя модулями сдвига $G_{12}, G_{23}, G_{13}$ и тремя коэффициентами Пуассона $v_{12}, v_{23}, v_{13}$. Из анализа литературных источников установлено, что при определении коэффициентов податливости пространственно-армированных композиционных материалов учитывается только объемное содержание и упругие характеристики армирующего материала. Исследования напряженно-деформированного состояния матрицы, модифицированной прошивочной нитью, показали, что на ее упругие характеристики кроме объемного содержания прошивочной нити оказывают влияние геометрические параметры прошивки, упругие характеристики пропитанной матричным материалом прошивочной нити, концентрация напряжений на ее границе. Это влияние имеет место при всех способах создания пространственных структур, образованных системой трех нитей. В статье также представлены результаты экспериментального определения упругих характеристик пространственно-армированных КМ и проведено их сравнение с теоретическими полученными компонентами тензора упругой податливости однонаправленно-армированного КМ.

Ключевые слова: композит, модифицированная матрица, прошивка, 3D структура.

\section{1. Введение}

Композиционные материалы (КМ) - это многокомпонентные материалы, состоящие из пластичной матрицы (основы), армированной наполнителями (волокнами, тканями и др.), обладающими высокой прочностью. Наибольшее распространение получили КМ с волокнами, уложенными в одной плоскости (2D структура) $[2,3,4,11,17]$.

Недостатками композиционных материалов с 2D структурами являются низкие физико-механические характеристики в 3-ем направлении, а также ярко выраженная анизотропия их свойств. Этих недостатков лишены композиционные материалы с 3D структурами, которые позволяют увеличить межслоевые прочностные и упругие характеристики.

Пространственные композиционные материалы могут быть образованы разными способами $[3,8]$. Первый способ основан на использовании системы двух нитей: искривленных нитей основы и прямолинейных нитей утка. Волокна основы пронизывают весь композиционный материал по всей толщине и соединяют лежащие рядом волокна утка по всей высоте либо через несколько слоев.

Второй способ, когда композиционные материалы, образованные системой трех нитей, имеют армирующие компоненты во всех трех направлениях выбранных осей координат. Армирующие волокна могут быть прямолинейны или иметь заданную степень искривления. Система трех нитей позволяет создавать армирующий каркас материала не только в декартовой, но и в цилиндрической системах координат. Одним из самых простых способов создания трехмерно армированных композиционных материалов является прошивка слоистых материалов (Рис. 1,1S, дополнительный материал). Другие способы создания пространственных КМ реализуется на специальных установках, соответствующих конфигурациям изделий (например, плетение трехмерных каркасов для теплозащитных покрытий) [2,3].

Основы теоретических расчетов пространственноармированных композиционных материалов приведены в работах $[1,2,3,7,8,14]$. При расчете упругих характеристик многослойных композиционных материалов используется принцип регуляризации структуры КМ [1-3,8-10]. В основу регуляризации положено разбиение многослойного композита на повторяющиеся элементы с последующим суммированием их упругих характеристик. В качестве повторяющегося элемента используется однонаправленный слой, состоящий из матрицы, армированной нитями прошивки (так называемая «модифицированная матрица») и армированной волокнами в плоскости слоя.

Разбиение на слои является чисто методическим приемом, упрощающим расчет упругих характеристик пространственно-армированного КМ, сведя его к слоистой модели $[6,7,8]$.

Прирасчетеупругогонапряженно-деформированного состояния однонаправленного слоя чаще всего его рассматривают как макроскопически однородную среду, состоящую из модифицированной матрицы (матрицы с характеристиками, эквивалентными характеристикам связующего, армированного прошивочной нитью) c распределенной в ней армирующей компонентой. Поэтому возникает необходимость установить соот-

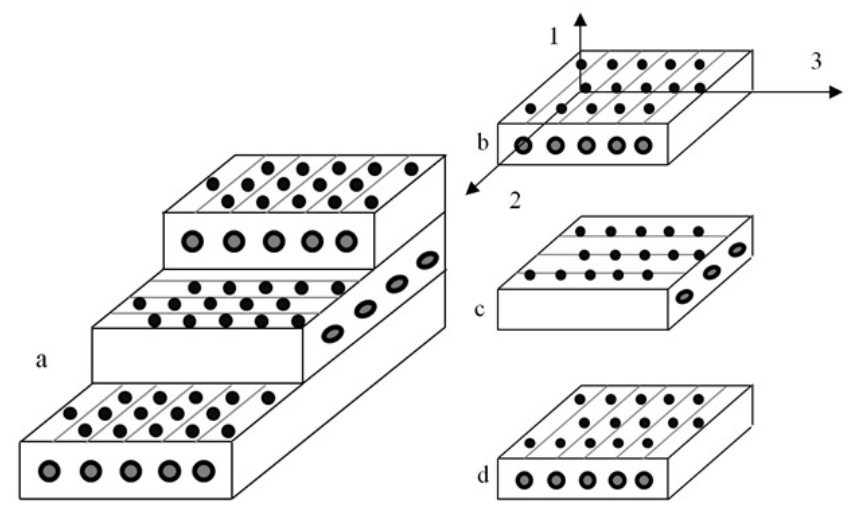

Рис. 1. Разбиение композиционного материала с 3D структурой на однонаправленные слои: многослойный композиционный материал с 3D структурой (поперечная прошивка) (a); однонаправленные слои, состоящие из модифицированной матрицы (прошитой нитями в направлении 1), армированной в направлении 2 (b, c, d)

Fig. 1. Decomposition of a composite materials with a 3D structure to unidirectional layers: multilayer composite material with a 3D structure (cross-stitching) (a); unidirectional layers consisting of a modified matrix (stitched in direction 1), reinforced in direction $2(b, c, d)$ 
ношения всех независимых компонент тензора податливости однонаправленного слоя в зависимости от упругих характеристик составляющих компонент, их объемного содержания и других макроскопических параметров, зависящих от способа создания пространственного композита.

Как показали исследования напряженно-деформированного состояния (НДС) модели матрицы с поперечной прошивкой на основе конечно-элементного анализа (см. далее) напряженное состояние модифицированной матрицы является неоднородным в связи с наличием концентраторов напряжений, вызванных прошивочной нитью.

Неоднородное распределение напряжений зависит от параметров прошивки, упругих характеристик пропитанной и отвержденной прошивочной нити, формы прошивочной нити. Учет этих факторов в опубликованных литературных источниках отсутствует. В зарубежной литературе также не найдены исследования учета вышеназванных факторов при определении упругих характеристик КМ с 3D структурами.

Научной новизной настоящей статьи является учет геометрических параметров прошивки, упругих характеристик пропитанной матричным материалом прошивочной нити, концентрации напряжений на ее границе через конечно-элементную модель модифицированной матрицы.

Задачами исследования явились:расчет характеристик модифицированной матрицы с учетом ее неоднородной структуры методом конечных элементов и расчет на этой основе упругих характеристик пространственноармированных композиционных материалов.

\section{2. Используемые модели и методы расчета}

Для композиционных полимерных материалов с пространственной структурой характерны существенные различия в значениях модулей упругости армирующей компоненты и связующего и высокое объемное заполнение армирующим материалом (40 - 70\%), что не позволяет использовать для определения их упругих характеристик традиционные для механики твердых деформируемых тел способов и методов их описания $[11,13]$. В работе [11] приведена схема аналитического расчета упругих характеристик композиционного материала методом разложения тензоров жесткости и податливости в ряд по объемным коэффициентам армирования. Этот метод допустимо использовать при малых (3-5\%) коэффициентах армирования. Коэффициент армирования при этом входит в расчетные выражения нелинейно, что приводит к трудностям реализации метода разложения. Следует учитывать также, что пространственное расположение волокон приводит к расчетной схеме в многосвязной области матрица-волокно, что создает большие трудности при решении задачи теории упругости по определению упругих характеристик KM с 3D структурой. Для композиционных материалов с высоким объемным содержанием армирующей компоненты расчет эффективных значений упругих характеристик компо- зиционного материала проводится на основе метода регуляризации его структуры $[3,5,7,8,14]$. Согласно этому методу упорядоченная реальная структура заменяется моделью, состоящей из периодически чередующихся однонаправленных слоев (Рис. 1).

Однонаправленный слой имеет девять коэффициентов податливости [5]

$$
\varepsilon_{i j}=a_{i j k l} \sigma_{k l}, i, j, k, l=1,2,3,
$$

$a_{i j k l}$ - компоненты тензора упругой податливости (формулы (29S) - (37S), дополнительный материал).

В приведенных выше методах расчета упругих характеристик модифицированной матрицы учитывается только объемное содержание армирующих волокон (прошивки) и механические характеристики прошивочной нити.

Теоретические методы определения компонент тензора податливости пространственно-армированных КМ в указанных выше работах базируются на следующих исходных предположениях:

1. Однонаправленно-армированный материал представляет собой сплошное маркоскопически однородное ортотропное тело.

2. Основной материал (в дальнейшем называемый матрицей) является упругим и анизотропным. Связь между деформациями и напряжениями в матрице и арматуре подчиняются закону Гука.

3. Между матрицей и арматурой существует полное сцепление.

4. Поперечно направленные к арматуре дополнительные напряжения, которые могут возникать при действии напряжений вдоль армирования в виду различных значений коэффициентов Пуассона для арматуры и матрицы, ничтожно малы.

5. При нагружении образца поперек направления армирования напряжения в матричном материале и арматуре одинаковы и равны усредненным напряжениям для всего объема рассматриваемого элемента.

Исследования проводились на однонаправленном углепластике на основе углеволокон УОЛ-300Р и фталонитрильного связующего PN-3M с поперечной прошивкой углеродной конструкционной нитью УКН-М-3К [12].

Механические характеристики углеволокон, фталонитрильного связующего и прошивочной нити представлены в Табл. 1S (дополнительный материал).

Аналитический метод определения коэффициентов податливости однонаправленного углепластика с прошивкой включает два этапа: 1) определение характеристик модифицированной (анизотропной) матрицы за счет осреднения ее упругих характеристик волокнами, уложенными в направлении, перпендикулярном плоскости композита; 2) расчет характеристик композита с модифицированной матрицей, армированного волокнами, параллельными плоскости композита (однонаправленный композит).

При определении компонентов тензора податливости модифицированной матрицы, в отличие от работ $[1-3,6-8]$, в данной работе была создана конечноэлементная модель (Рис. 2S, дополнительный материал), учитывающая геометрические параметры прошивки 
и механические характеристики прошивочной нити, концентрацию напряжений на границе прошивочной нити (Табл. 2S, дополнительный материал).

При определении усредненных упругих деформационных характеристик композита на основе конечно-элементной модели используются усредненные напряжения для всего объема представительного элемента с учетом параметров прошивки, механических характеристик прошивочной нити и концентраторов напряжений.

\section{3. Результаты расчетов}

\section{1. Исследование упругих характеристик модибицированной матрищь}

На Рис. 2 представлена картина напряженнодеформированного состояния «модифицированной» матрицы с прошивкой, из которой видно наличие концентрации напряжений на границе прошивочной нити и полимерного связующего. Общий вид картины напряженно-деформированного состояния модифицированной матрицы представлен на Рис. 3S (дополнительный материал).

Аналитически определенные упругие характеристики модифицированной матрицы согласно зависимостям ((1S)-(37S), дополнительный материал) [1-3,6-8], определенные только с учетом объемного содержания прошивочной нити, представлены в Табл. 1. Также в Табл. 1 представлены упругие характеристики модифицированной матрицы, полученные на основе конечноэлементной модели (КЭМ) с учетом механико-геометрических параметров прошивки.

На основе анализа НДС модели модифицированной матрицы были определены ее упругие характери-

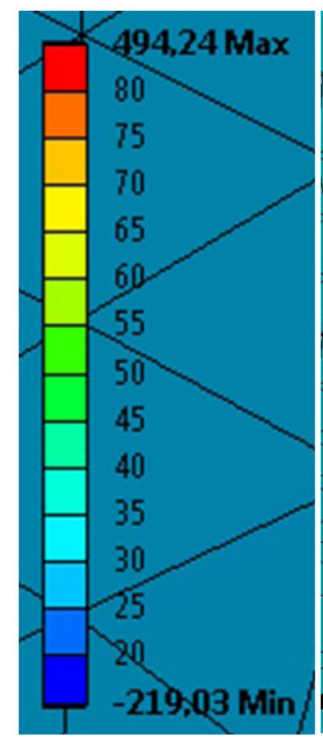

a

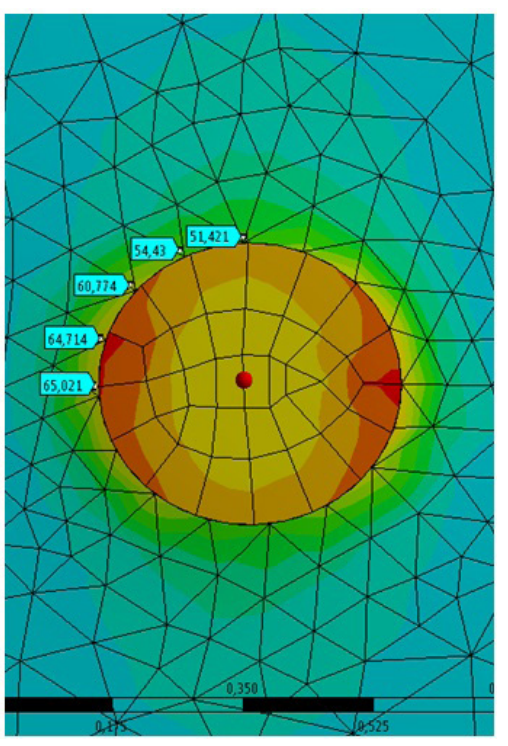

b
Puc. 2. (Color online) Шкала градации напряжений в МПа (а) и картина напряженно-деформированного состояния «модифицированной» матрицы с прошивкой с зонами концентрации напряжений (b).

Fig. 2. (Color online) Stress gradation scale, MPa (a) and the stressstrain state of the "modified" matrix with piercing and stress concentration zones (b). стики путем осреднения по объему представительного элемента согласно [7] (Табл. 3S, дополнительный материал).

Сравнивая значения упругих характеристик, вычисленных только с учетом объемного содержания, c данными, полученными по конечно-элементной модели с учетом параметров прошивки, видим, что в результате наличия концентраторов напряжений по границе «связующее-волокно» упругие характеристики «модифицированной» матрицы в целом уменьшаются.

\section{2. Расчет характеристик 3D композита}

Сравнение упругих характеристик однонаправленного композита на основе модифицированной матрицы без учета параметров прошивки и полученных на основе КЭМ представлено в Табл. 2.

Из Табл. 2 видно, что упругие характеристики, полученные по КЭМ, в силу наличия концентраторов напряжений имеют заниженные значения по отношению к методикам, указанным в работах [1-3].

Наибольшее влияние прошивки связано с увеличением прочности однонаправленного слоя в направлении прошивки. Согласно расчетам $[11,13,16]$, прочность однонаправленного слоя в направлении прошивки 1 возрастает на 75\% по сравнению с непрошитым однонаправленным слоем.

\section{4. Экспериментальные исследования}

Экспериментальное определение упругих свойств однонаправленного композита с прошивкой проводились на образцах с размерами $250 \times 20 \times 2$ мм.

Табл. 1. Сравнение упругих характеристик модифицированной матрицы, вычисленных аналитически, с полученными на основе конечно-элементной модели.

Table 1. Comparison of the modified matrix elastic characteristics, calculated analytically, with those obtained on the finite element model (FEM) basis.

\begin{tabular}{|c|c|c|}
\hline $\begin{array}{c}\text { Характеристика/ } \\
\text { Characteristic }\end{array}$ & $\begin{array}{c}\text { Полученная по } \\
\text { аналитическим } \\
\text { зависимостям/ } \\
\text { Obtained from } \\
\text { analytical } \\
\text { dependencies }\end{array}$ & $\begin{array}{c}\text { Полученная на } \\
\text { основе КЭМ/ } \\
\text { Obtained on the } \\
\text { FEM basis }\end{array}$ \\
\hline$E_{1}$, ГПа $/ E_{1}, \mathrm{GPa}^{*}$ & 6.7 & 5.4 \\
\hline$E_{2}, \Gamma \Pi а / E_{2}, \mathrm{GPa}$ & 5.0 & 4.8 \\
\hline$E_{3}, \Gamma \Pi \mathrm{a} / E_{3}, \mathrm{GPa}$ & 5.0 & 4.8 \\
\hline$v_{12}$ & 0.338 & 0.29 \\
\hline$v_{23}$ & 0.386 & 0.40 \\
\hline$v_{13}$ & 0.338 & 0.29 \\
\hline$G_{12}$, ГПа $/ G_{12}, \mathrm{GPa}$ & 1.82 & 1.8 \\
\hline$G_{23}, \Gamma \Pi a / G_{23}, \mathrm{GPa}$ & 1.81 & 1.7 \\
\hline$G_{13}, \Gamma \Pi а / G_{13}, \mathrm{GPa}$ & 1.82 & 1.8 \\
\hline
\end{tabular}

*здесь и далее: 1 - направление прошивки 
табл. 2. Сравнение упругих характеристик однонаправленного композита на основе модифицированной матрицы, вычисленные аналитически и на основе КЭМ.

Table 2. Comparison of the elastic characteristics of a unidirectional composite based on a modified matrix, calculated analytically, and based on FEM.

\begin{tabular}{|c|c|c|}
\hline $\begin{array}{c}\text { Характеристика } \\
\text { Characteristic }\end{array}$ & $\begin{array}{c}\text { Без учета } \\
\text { параметров } \\
\text { прошивки/ } \\
\text { Without taking } \\
\text { into account } \\
\text { piercing parameters }\end{array}$ & $\begin{array}{c}\text { С учетом } \\
\text { параметров } \\
\text { прошивки/ } \\
\text { With taking into } \\
\text { account piercing } \\
\text { parameters }\end{array}$ \\
\hline$E_{1}, \Gamma \Pi а / E_{1}, \mathrm{GPa}^{*}$ & 14.8 & 7.3 \\
\hline$E_{2}, \Gamma \Pi \mathrm{a} / E_{2}, \mathrm{GPa}$ & 128.0 & 127.9 \\
\hline$E_{3}, \Gamma \Pi а / E_{3}, \mathrm{GPa}$ & 14.0 & 13.5 \\
\hline$v_{12}$ & 0.02 & 0.011 \\
\hline$v_{23}$ & 0.24 & 0.24 \\
\hline$v_{13}$ & 0.49 & 0.29 \\
\hline$G_{12}, \Gamma \Pi a / G_{12}, \mathrm{GPa}$ & 4.7 & 4.7 \\
\hline$G_{23}, \Gamma \Pi a / G_{23}, \mathrm{GPa}$ & 4.7 & 4.5 \\
\hline$G_{13}, \Gamma \Pi a / G_{13}, \mathrm{GPa}$ & 4.4 & 1.8 \\
\hline$\mu_{2}$ & 0.6 & 0.6 \\
\hline
\end{tabular}

*здесь и далее: 2 - направление основного армирования

Этот раздел включает следующие испытания образцов с прошивкой и без прошивки: 1) испытания на растяжение: определение продольного и поперечного модулей упругости, коэффициентов Пуассона; 2) испытания на трехточечный изгиб: определение модуля упругости при изгибе, определение межслойного модуля упругости, определение предела прочности при изгибе; 3) испытания на прочность при межслойном сдвиге.

Определение модуля межслойного сдвига проводилось по результатам испытаний на изгиб по методике [6]. Испытание на прочность при сдвиге проводили на образцах (Рис. 4S, дополнительный материал) в соответствии со стандартом ASTM D 2345-65 Т. Испытания на растяжение проводились на универсальных испытательных машинах Instron 3382 (Германия, предельное усилие $100 \mathrm{kH}$ ) и Zwick/Roell Z010 (ГДР, предельное усилие $10 \mathrm{kH}$ ); на изгиб на установках Zwick/Roell Z010 с изгибным реверсом и AS-102 (Plastic Bending Tester, Венгрия); прочность при сдвиге на машинах Zwick/Roell Z010 и FP 10 ("Fritz Heckert", ГДР).

Сравнение теоретически определенных значений упругих характеристик однонаправленно-армированного композита с модифицированной матрицей с экспериментальными значениями представлено в Табл. 3. Результаты испытаний образцов с наличием прошивки и без представлены в Табл. $3 \mathrm{~S}$ (дополнительный материал).

Анализ полученных результатов позволяет сделать следующие выводы:

- поперечная прошивка увеличила поперечный модуль упругости однонаправленного слоя на $32 \%$ по сравнению с непрошитым;

- модуль межслойного сдвига благодаря прошивке увеличился на $12 \%$; табл. 3. Сравнение теоретически определенных значений упругих характеристик однонаправленно-армированного композита с модифицированной матрицей с экспериментальными значениями.

Table 3. Comparison of the elastic characteristics theoretically determined values of a unidirectionally reinforced composite with a modified matrix with experimental values.

\begin{tabular}{|c|c|c|}
\hline $\begin{array}{c}\text { Характеристика/ } \\
\text { Characteristic }\end{array}$ & $\begin{array}{c}\text { Теоретически } \\
\text { определенное } \\
\text { значения/ } \\
\text { Theoretically } \\
\text { determined values }\end{array}$ & $\begin{array}{c}\text { Экспериментально } \\
\text { полученное } \\
\text { значения/ } \\
\text { Experimentally } \\
\text { obtained values }\end{array}$ \\
\hline$E_{1}, \Gamma \Pi \mathrm{a} / E_{1}, \mathrm{GPa}^{*}$ & 14.8 & - \\
\hline$E_{2}, \Gamma \Pi а / E_{2}, \mathrm{GPa}$ & 127.9 & 125.4 \\
\hline$E_{3}, \Gamma \Pi \mathrm{a} / E_{3}, \mathrm{GPa}$ & 13.5 & 6.9 \\
\hline$v_{12}$ & 0.02 & - \\
\hline$v_{23}$ & 0.24 & 0.36 \\
\hline$v_{13}$ & 0.49 & - \\
\hline$G_{12}, \Gamma \Pi а / G_{12}, \mathrm{GPa}$ & 4.7 & 1.55 \\
\hline$G_{23}, \Gamma \Pi a / G_{23}, \mathrm{GPa}$ & 4.5 & 5.9 \\
\hline$G_{13}, \Gamma \Pi a / G_{13}, \mathrm{GPa}$ & 4.4 & - \\
\hline
\end{tabular}

- повысилась прочность в направлении прошивки на $75 \%$;

- продольный модуль упругости снизился на 26\%;

- модуль упругости при изгибе снизился на $52 \%$;

- прочность при растяжении вдоль основного армирования снизилась на $35 \%$ по сравнению с непрошитым.

Снижение упругих и прочностных характеристик однонаправленного слоя вдоль основного армирования произошло, по нашему мнению, по следующим причинам: 1) в направлении основного армирования прошивка вызывает концентрацию напряжений и ослабление адгезионной прочности прошивочной нити с матричным материалом, что вызывает снижение адгезионной прочности нитей основного направления с модифицированной матрицей; 2) прошивка однаправленного слоя производилась до пропитки, в связи с этим имеет место повреждение волокон основного армирования.

\section{5. Выводы}

Основным преимуществом 3D армированных структур с прошивкой является увеличение прочностных (сопротивлению расслоению) и упругих (сопротивление межслойному сдвигу) характеристик в направлении прошивки.

Одновременно с увеличением указанных выше характеристик прошивка снижает модули упругости при растяжении и изгибе, прочность при растяжении, изгибе и сдвиге вдоль основного армирования однонаправленного слоя.

Для объективной оценки напряженно-деформированного состояния композитов с прошивкой необходимо учитывать геометрические и механические параметры прошивки. 
Дополнительньй материал/Supplementary Material. Электронная версия статьи содержит дополнительный материал, доступный безвозмездно на сайте журнала (lettersonmaterials.com)./The online version of this paper contains supplementary material available free of charge at the journal's Web site (lettersonmaterials.com).

Благодарности/Aknowledgements. Исследование выполнено при бинансовой поддержке РФФИ и Правительства Республики Башкортостан в рамках научного проeкma № 17-48-020978 p_a./The study was carried out with the financial support of the RFRF and the Government of the Republic of Bashkortostan within the confines of the research project No. 17-48-020978 p_a.

\section{Литература/References}

1. A.K. Malmeyster, V.P. Tamuzh, G.A. Teters. Soprotivleniye zhestkikh polimernykh materialov. Riga, Zinatne (1972) 500 p. (in Russian) [А.К. Малмейстер, В.П. Тамуж, Г.А. Тетерс. Сопротивление жестких полимерных материалов. Рига, Зинатне (1972) 500 с.]

2. I. G. Zhigun, V.A. Polyakov. Svoystva prostranstvennoarmirovannykh plastikov. Ed. by Y.M. Tarnopolskiy. Riga, Zinatne (1978) 215 p. (in Russian) [И.Г. Жигун, B. А. Поляков. Свойства пространственно-армированных пластиков. Под ред. Ю.М. Тарнопольского. Рига, Зинатне (1978) 215 с.]

3. Y.M. Tarnopolskiy, I.G. Zhigun, V.A. Polyakov. Prostranstvenno-armirovannyye kompozitsionnyye materialy. Spravochnik. Moscow, Mashinostroyeniye (1987) 224 p. (in Russian) [Ю.М. Тарнопольский, И.Г. Жигун, В.А. Поляков. Пространственноармированные композиционные материалы. Справочник. Москва, Машиностроение (1987) 224 с.]

4. S. G. Lekhnitskiy. Teoriya uprugosti anizotropnogo tela. 2nd edition. Moscow, Nauka (1977) 416 p. (in Russian) [C.Г. Лехницкий. Теория упругости анизотропного тела. Изд. 2-е. Москва, Наука (1977) 416 с.]

5. Y.M. Tarnopolskiy, T.Y. Kintsis. Metody staticheskikh ispytaniy armirovannykh plastikov. 3rd edition. Moscow, Khimiya (1981) 270 p. (in Russian) [Ю.М. Тарнопольский, Т. Я. Кинцис. Методы статических испытаний армированных пластиков. Изд. 3-е. Москва, Химия (1981) 270 с.]

6. B.A. Lyukshin. Kompozitsionnyye materialy. Tomsk, Tomsk State University of Control Systems and Radioelectronics (2012) 101 p. (in Russian) [Б. А. Люкшин. Композиционные материалы. Томск,
Томский государственный университет систем управления и радиоэлектроники (2012) 101 с.]

7. D.S. Abolinsh. Mekhanika polimerov. 4, 47 (1965) (in Russian) [Д. С. Аболиньш. Механика полимеров, 4, 47 (1965).]

8. A.M. Skudra, F.Y. Bulavs, K. A. Rotsens. Polzuchest i staticheskaya ustalost armirovannykh plastikov. Riga, Zinatne (1971) 238 p. (in Russian) [A. М. Скудра, Ф.Я. Булавс, К.А. Роценс. Ползучесть и статическая усталость армированных пластиков. Рига, Зинатне (1971) 238 c.]

9. L. Brautman, R. Krok. Kompozitsionnyye materialy. Tom 5. Razrusheniye i ustalost'. Translated and edited by G.P. Cherepanova. Moscow, Mir (1978) 488 p. (in Russian) [Л. Браутман, Р. Крок. Композиционные материалы. Том 5. Разрушение и усталость. Перевод с англ. под ред. Г. П. Черепанова. Москва, Мир (1978) 488 c.]

10. G.A. Molodtsov, V.Y. Bitkin, V.F. Silyonov, F.F. Urmanov. Formostabilnyye i intellektualnyye konstruktsii iz kompozitsionnykh materialov. Moscow, Mashinostroyeniye (2000) 352 p. (in Russian) [Г.А. Молодцов, В.Е. Биткин, В.Ф. Сильонов, Ф.Ф. Урманов. Формостабильные и интеллектуальные конструкции из композиционных материалов. Москва, Машиностроение (2000) 352 с.]

11. V. S. Ivanova, I. M. Kopyev, L. R. Botvina, T. D. Shermergor. Uprochneniye metallov voloknami. Moscow, Nauka (1973) 206 p. (in Russian) [В. С. Иванова, И. М. Копьев, Л. Р. Ботвина, Т. Д. Шермергор. Упрочнение металлов волокнами. Москва, Наука (1973) 206 с.]

12. V.S. Zhernakov, Y.S. Pervushin, P.V. Solovyev. Vestnik UGATU. 21 (3), 19 (2017) (in Russian) [B. С. Жернаков, Ю. С. Первушин, П. В. Соловьев. Вестник УГАТУ. 21 (3), 19 (2017).]

13. G. A. Van Fo Fy. Osnovy teorii polimernykh tel s oriyentirovannoy strukturoy. Abstract of doc. diss. Kiev (1965) 10 p. (in Russian) [Г. А. Ван Фо Фы. Основы теории полимерных тел с ориентированной структурой. Автореферат докт. дисс. Киев (1965) 10с.]

14. V. V. Bolotin. Mekhanika polimerov. 2, 27 (1965) (in Russian) [В.В. Болотин. Механика полимеров. 2, 27 (1965).]

15. S.W. Tsai. Structural behavior of composite materials. NASA CR-71 (1964).

16. W. Voigt. Lehrbuch der Kristallphysik. Berlin-Liepzig, Teubner-Verlag (1910).

17. R. G. Hill. J. Mech. Phys. Solids. 13 (4), 213 (1965). Crossref 